\title{
Simplicillium lanosoniveum, a hyperparasite on Aecidium elaeagni-latifoliae in India
}

\author{
Pankaj Baiswar • S. V. Ngachan • H. Rymbai • \\ Satish Chandra
}

Received: 16 April 2014 / Accepted: 5 August 2014 / Published online: 15 August 2014

(C) Australasian Plant Pathology Society Inc. 2014

\begin{abstract}
A hyperparasite was observed on Aecidium elaeagni-latifoliae during a survey in Umiam, Meghalaya. Morphological characterization using light, scanning electron microscopy and molecular characterization by sequencing ITS region, large subunit of nuclear ribosomal DNA and phylogenetic analysis revealed the identity of the pathogen as Simplicillium lanosoniveum. This is the first record of hyperparasite S. lanosoniveum on A. elaeagni-latifoliae from India.
\end{abstract}

Keywords Simplicillium · Elaeagnus $\cdot$ ITS $\cdot$ LSU $\cdot$ Aecidium

Elaeagnus latifolia is an evergreen woody shrub, common in subtropical and temperate areas and belongs to family Elaeagnaceae. It is mostly grown in semi-wild conditions, fruits are locally known as Soh-Shang and are rich in vitamins $\mathrm{A}, \mathrm{C}$ and $\mathrm{E}$ and contain several compounds which have anticancerous properties. It is considered as having great potential in this region because of its hardy nature and early bearing (Patel et al. 2008).

Rust of E. latifolia caused by Aecidium elaeagnilatifoliae is an emerging disease in this region. During a regular survey we came across hyperparasitised pustules of rust on the leaves of E. latifolia (Fig. 1). Microscopic examination was conducted using light and scanning electron microscopy. Molecular analysis was also done for confirmation. Light microscopy was conducted using an Olympus BX 53 microscope equipped with a digital camera DP 72, Olympus, and image analysis softwarecellSens Standard 1.5, Olympus. Observations were made

P. Baiswar $(\bowtie) \cdot$ S. V. Ngachan $\cdot$ H. Rymbai $\cdot$ S. Chandra

ICAR Research Complex for NEH Region, Umiam 793

103, Meghalaya, India

e-mail: pbaiswar@yahoo.com using $3 \%$ potassium hydroxide as mounting medium. Hyperparsitised pustules were selected using a dissecting microscope and placed on double-sided cellotape then sputter-coated with gold under vacuum using Fine Coat Ion Sputter JFC-1100. Gold-coated samples were then placed on aluminium stubs for scanning electron microscopy (SEM) (JEOL JSM 6360, JEOL, Tokyo, Japan). A voucher specimen has been deposited in Ajrekar Mycological Herbarium (AMH- 9654). Microscopic analysis using light and scanning electron microscopy clearly indicated the affinity of the fungus with Simplicillium lanosoniveum. Phialides were solitary and conidial dimensions were $2.53 .5 \times 12 \mu \mathrm{m}$ (Zare and Gams 2004). SEM also revealed, whole rust pustules were covered with the growth of the hyperparasite (Fig 2, 3, 4).

Nested PCR was done using NL1 and LR5 for the first round then NL1 and LR3 for second round for amplification of large subunit (LSU) of nrDNA containing D1 and D2 domains (Vilgalys and Hester 1990). The ITS region comprising ITS1-5.8S-ITS2 was amplified using primers ITS1F and ITS4 (Gardes and Bruns 1993; White et al. 1990). PCR was done using initial denaturation for $5 \mathrm{~min}\left(94^{\circ} \mathrm{C}\right)$, denaturation 30 s $\left(94{ }^{\circ} \mathrm{C}\right)$, annealing 40 s $\left(54-50{ }^{\circ} \mathrm{C}\right.$, touchdown with $1{ }^{\circ} \mathrm{C}$ decrement in every cycle), extension 1:10s or 50s (depending on the primer combination) $\left(72{ }^{\circ} \mathrm{C}\right)$ and final extension $10 \mathrm{~min}$ $\left(72{ }^{\circ} \mathrm{C}\right)(35$ cycles $)$. Sequencing was done using ITS1F and 4 for ITS region and NL1 and LR3 for LSU region. The sequences of ITS (KJ408447) and LSU (KJ135022) obtained in this study have been deposited in GenBank. Similarity checks were done at NCBI website. For further analysis, sequences of LSU and ITS of closely related sequences were downloaded from NCBI (Table 1,2). Majority of the sequences included in the analysis were from Nonaka et al. (2013a) and Sung et al. (2001). Alignment was done using Clustal W implemented in MEGA 5.0 (Tamura et al. 2011 and references therein). Model 


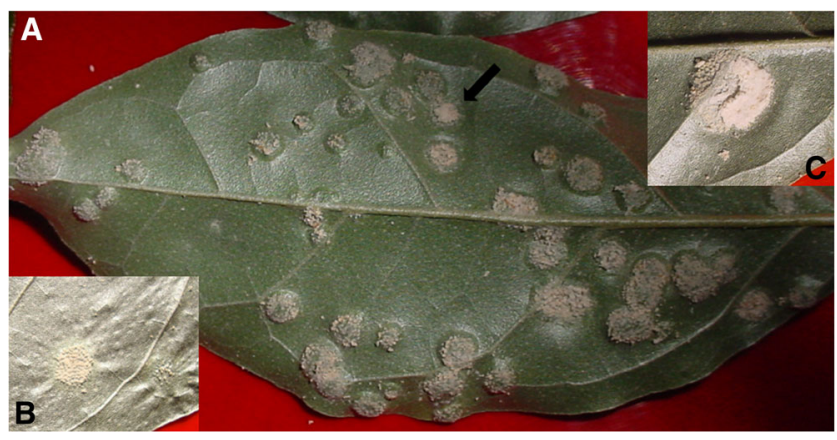

Fig. 1 Parasitized and nonparasitized rust pustules on Elaeagnus latifolia

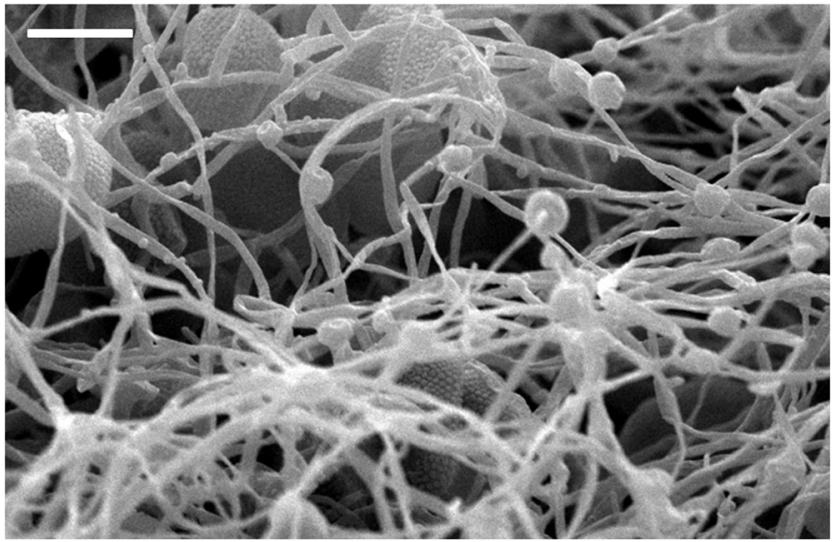

Fig. 2 Rust pustules covered with hyperparasite observed using SEM $(\mathrm{Bar}=10 \mu \mathrm{m})$

selection was done using jModeltest (Posada 2008). Phylogenetic analysis was done using MrBayes v.3.1.2 (Huelsenbeck and Ronquist 2001). Parameters used were generations $=$ $2,000,000$, sampling frequency $=100, \mathrm{nst}=6$, rates $=$ gamma, no of chains set to 4 with two simultaneous runs and burnin= 5,000 . Tracer was used for convergence analysis (Rambaut and Drummond 2007). FigTree was used for visualising the tree (Rambaut 2012).

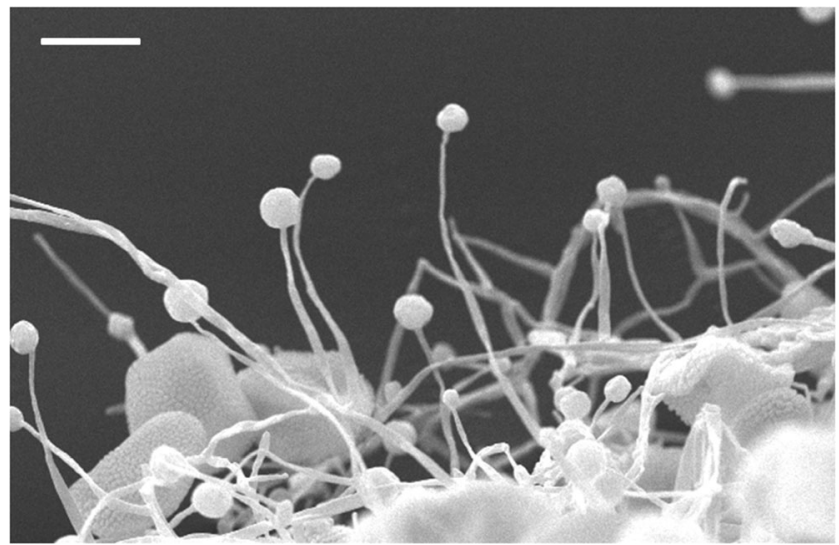

Fig. 3 Solitary phialides of Simplicillium lanosoniveum bearing conidia $($ Bar $=10 \mu \mathrm{m})$

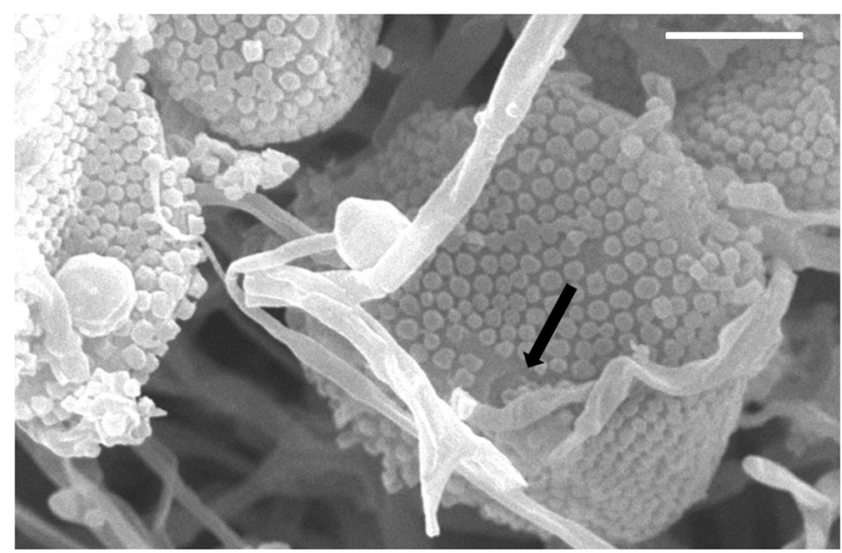

Fig. 4 Parasitized aeciospore of Aecidium elaeagni-latifoliae (Bar= $5 \mu \mathrm{m})$

Bayesian analysis with LSU sequences recovered the fungus in a well-supported (posterior probability $=1.0$ ) clade with species of Simplicillium. (Fig. 5). The sequence of Verticillium dahliae (VDU17425) was used as root. Species determination

Table 1 Large subunit sequences obtained from NCBI

\begin{tabular}{|c|c|c|c|}
\hline Organism & $\begin{array}{l}\text { LSU } \\
\text { sequence }\end{array}$ & Strain & References \\
\hline Simplicillium chinense & JQ410322 & LC1345 & Liu and Cai 2012 \\
\hline Simplicillium chinense & JQ410321 & LC1342 & Liu and Cai 2012 \\
\hline Simplicillium lanosoniveum & КC009174 & 41559-3 & $\begin{array}{l}\text { Rajkumar and Chaturvedi } \\
2012 \text { (Unpublished) }\end{array}$ \\
\hline Simplicillium obclavatum & JX081385 & BAB519_asp18 & Raol et al. 2012 (Unpublished) \\
\hline Simplicillium obclavatum & HQ232175 & CBS 311.74 & Summerbell et al. 2011 \\
\hline Simplicillium lanosoniveum & HQ232006 & CBS 321.72 & Summerbell et al. 2011 \\
\hline Simplicillium lanosoniveum & AF339553 & CBS 704.86 & Sung et al. 2001 \\
\hline Simplicillium lanosoniveum & AF339554 & IMI 317442 & Sung et al. 2001 \\
\hline Simplicillium lamellicola & AF339552 & CBS 116.25 & Sung et al. 2001 \\
\hline Simplicillium obclavatum & AF339517 & CBS 311.74 & Sung et al. 2001 \\
\hline Pochonia chlamydosporia & AF339544 & CBS 504.66 & Sung et al. 2001 \\
\hline Lecanicillium lecanii & AF339556 & CBS 126.27 & Sung et al. 2001 \\
\hline Rotiferophthora angustispora & AF339535 & CBS 101437 & Sung et al. 2001 \\
\hline Pochonia rubescens & AB709833 & FKI-5829 & Nonaka et al. 2013b \\
\hline Pochonia rubescens & AB709832 & FKI-1083 & Nonaka et al. 2013b \\
\hline Pochonia bulbillosa & AB709809 & FKI-4395 & Nonaka et al. 2013b \\
\hline Haptocillium sinense & AF339546 & CBS 131.95 & Sung et al. 2001 \\
\hline Haptocillium sinense & AF339545 & CBS 567.95 & Sung et al. 2001 \\
\hline Haptocillium sinense & AF339543 & IMI 356051 & Sung et al. 2001 \\
\hline Haptocillium sphaerosporum & AF339541 & CBS 522.80 & Sung et al. 2001 \\
\hline Lecanicillium primulinum & AB712265 & JCM 18527 & Kaifuchi et al. 2013 \\
\hline Lecanicillium primulinum & AB712264 & JCM 18526 & Kaifuchi et al. 2013 \\
\hline Lecanicillium attenuatum & AF339565 & CBS 402.78 & Sung et al. 2001 \\
\hline Lecanicillium psalliotae & AF339560 & CBS 532.81 & Sung et al. 2001 \\
\hline Verticillium dahliae & VDU17425 & ATCC 16535 & Rehner and Samuels 1995 \\
\hline Verticillium nigrescens & EF543841 & CBS 577.50 & Zare et al. 2007 \\
\hline Simplicillium lanosoniveum & HQ232006 & CBS 321.72 & Summerbell et al. 2011 \\
\hline Simplicillium lanosoniveum & AF339554 & IMI 317442 & Sung et al. 2001 \\
\hline Simplicillium obclavatum & HQ232175 & CBS 311.74 & Summerbell et al. 2011 \\
\hline Simplicillium lamellicola & AF339552 & CBS 116.25 & Sung et al. 2001 \\
\hline
\end{tabular}


Table 2 ITS sequences obtained from NCBI

\begin{tabular}{|c|c|c|c|c|}
\hline Organism & ITS sequence & Strain & References & Source \\
\hline Simplicillium lanosoniveum & EF641862 & CBS 962.72 & Zare and Gams 2008 & - \\
\hline Simplicillium cylindrosporum & AB604006 & FKI-5985 & Nonaka et al. 2013a & soil \\
\hline Simplicillium lanosoniveum & AJ292396 & CBS 704.86 & Zare et al. 2000 & - \\
\hline Simplicillium subtropicum & AB604001 & JCM 18183 & Nonaka et al. 2013a & soil \\
\hline Simplicillium minatense & AB603991 & JCM 18177 & Nonaka et al. 2013a & soil \\
\hline Simplicillium obclavatum & AB604000 & JCM 18179 & Nonaka et al. 2013a & soil \\
\hline Simplicillium lanosoniveum & EU939525 & $\mathrm{Cs} 0701$ & Chen et al. 2008 & $\begin{array}{l}\text { Salvinia molesta } \\
\quad \text { (causing brown spot) }\end{array}$ \\
\hline Simplicillium lanosoniveum & EF513003 & IMI 303103b & Kouvelis et al. 2008 & - \\
\hline Acremonium obclavatum & AJ292394 & CBS 311.74 & Zare et al. 2000 & - \\
\hline Simplicillium minatense & AB603992 & Type strain & Nonaka et al. 2013a & Soil \\
\hline Torrubiella luteorostrata & AY624174 & CBS 398.86 & Luangsaard et al. 2005 & - \\
\hline Verticillium chlamydosporium var chlamydosporium & AJ292397 & CBS 103.65 & Zare et al. 2000 & - \\
\hline Simplicillium subtropicum & AB603995 & JCM 18181 & Nonaka et al. 2013a & Soil \\
\hline Simplicillium subtropicum & AB603990 & JCM 18180 & Nonaka et al. 2013a & Soil \\
\hline Simplicillium aogashimaense & AB604004 & JCM 18168 & Nonaka et al. 2013a & Soil \\
\hline Simplicillium aogashimaense & AB604002 & JCM 18167 & Nonaka et al. 2013a & Soil \\
\hline Simplicillium sympodiophorum & AB604003 & JCM 18184 & Nonaka et al. 2013a & Soil \\
\hline Simplicillium lamellicola & AB378533 & KYK00006 & $\begin{array}{l}\text { Ilyas et al. } 2008 \\
\text { (Unpublished) }\end{array}$ & Coccidae, Hemiptera \\
\hline Simplicillium lamellicola & AJ292393 & CBS 116.25 & Zare et al. 2000 & - \\
\hline Simplicillium chinense & JQ410324 & LC1345 & Liu and Cai 2012 & - \\
\hline Simplicillium chinense & JQ410323 & LC1342 & Liu and Cai 2012 & - \\
\hline Simplicillium wallacei & EF513022 & IMI 331549 & Kouvelis et al. 2008 & - \\
\hline Simplicillium wallacei & EF641891 & CBS 101237 & Zare and Gams 2008 & Lepidopteran larva on palm leaf \\
\hline
\end{tabular}

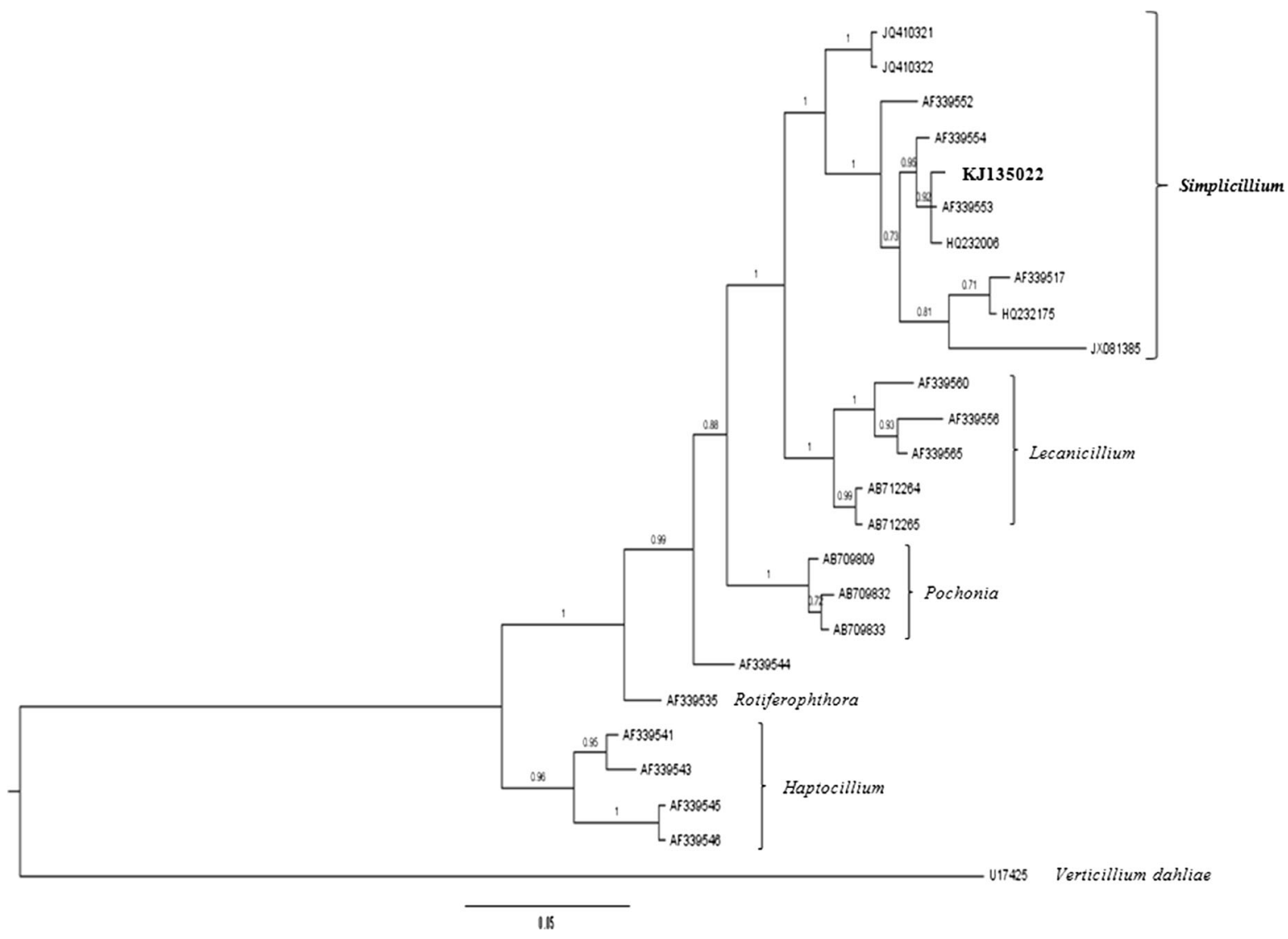

Fig. 5 Phylogenetic analysis based on large subunit sequences 


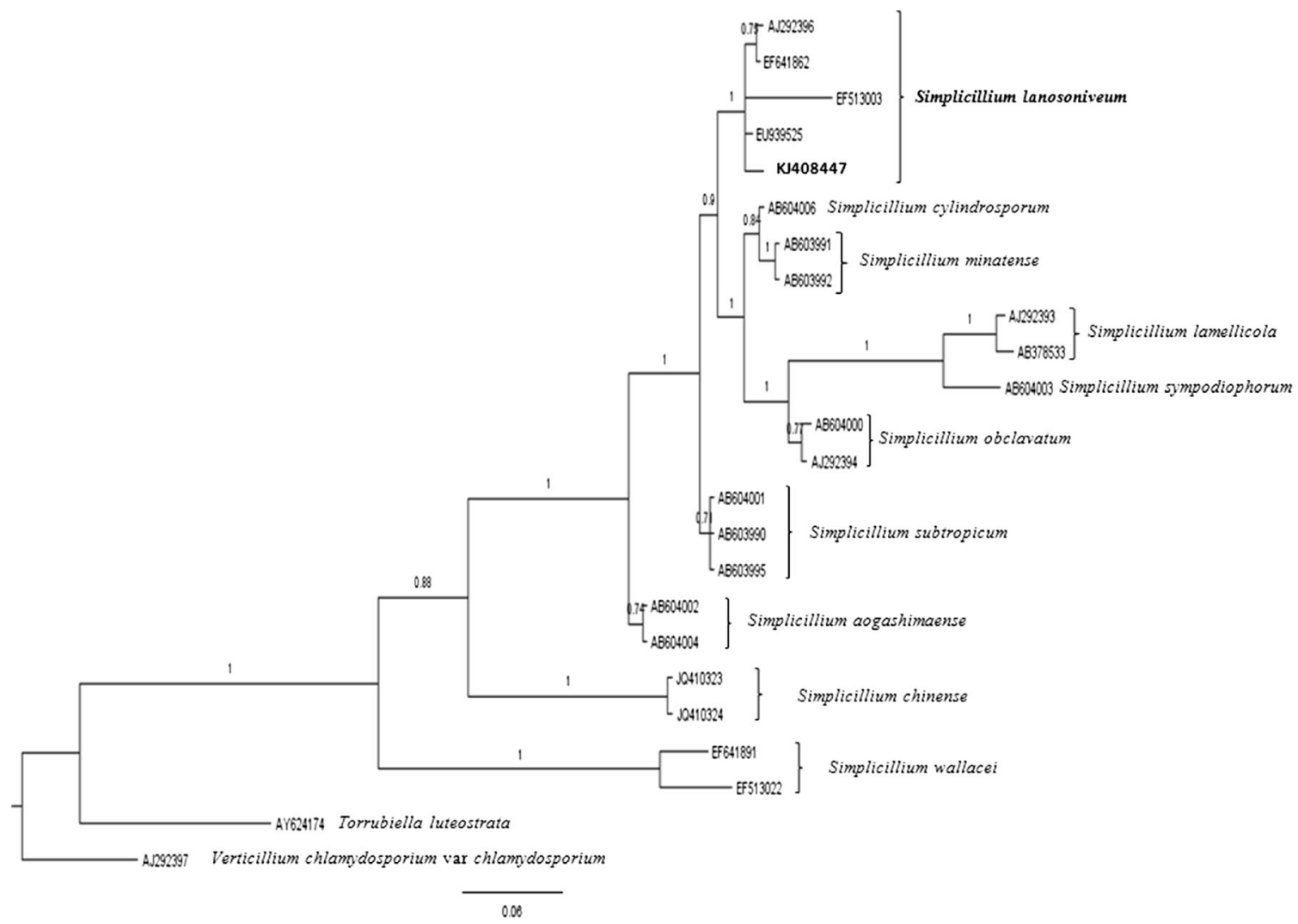

Fig. 6 Phylogenetic analysis based on ITS region

was done by conducting phylogenetic analysis using ITS region and Verticillium chlamydosporium var. chlamydosporium (=Pochonia chlamydosporia var. chlamydosporia) (AJ292397) sequence was chosen as outgroup. Results clearly indicated that the fungus belongs to $S$. lanosonieveum since it clustered with $S$. lanosonieveum with posterior probability value of 1.0 (Fig. 6). For ITS region minimum evolution method as implemented in MEGA 5.0 (Tamura et al. 2011 and references therein) was also used and it also clustered our sequence with $S$. lanosoniveum with bootstrap support value of $98 \%$.

Verticillium was regarded as heterogeneous group having phytopathogenic, fungicolous, entomopathogenic and saprophytic species. The genus was divided into sections Verticillium, Nigrescentia, Prostrata and Albo-erecta. Fungicolous and enomopathogenic species of Verticilillium were placed in the genera Simplicillium and Lecanicillium based on a systematic study by Zare and Gams (2004). A phylogenetic concordance method is used for species delimitation in these genera.

So, to our knowledge this is the first record of $S$. lanosoniveum on A. elaeagni-latifoliae causing rust of E. latifolia in India. Simplicillium lanosoniveum has a potential to be used for managing this rust as a sustainable and ecofriendly alternative.
Acknowledgements Authors would like to thank Animal Production including Poultry Science of our Institute for providing facilities for molecular work and to the Head of SAIF, Dr Sudeep Dey (Scientific Officer), Dr R. Charkraborty, N. K. Rynjah for scanning electron microscopy at North-Eastern Hill University, Shillong, Meghalaya, India. Microscope used in this study has been procured under National Initiative on Climate Resilient Agriculture project (PI, Dr DJ Rajkhowa).

\section{References}

Chen RS, Huang CC, Li JC, Tsay GJ (2008) First report of Simplicillium lanosoniveum causing brown spot on Salvinia auriculata and S. molesta in Taiwan. P1 Dis 92:1589

Gardes M, Bruns TD (1993) ITS primers with enhanced specificity for basidiomycetes - application to the identification of mycorrhizae and rusts. Mol Ecol 2:113-118

Huelsenbeck JP, Ronquist F (2001) MRBAYES: Bayesian inference of phylogenetic trees. Bioinformatics 17:754-755

Kaifuchi S, Nonaka K, Mori M, Shiomi K, Omura S, Masuma R (2013) Lecanicillium primulinum, a new hyphomycete (Cordycipitaceae) from soils in the Okinawa's main island and the Bonin Islands, Japan. Mycoscience 54:291-296

Kouvelis VN, Sialakouma A, Typas MA (2008) Mitochondrial gene sequences alone or combined with ITS region sequences provide firm molecular criteria for the classification of Lecanicillium species. Mycol Res 112:829-844

Liu F, Cai L (2012) Morphological and molecular characterization of a novel species of Simplicillium from China. Cryptog Mycolog 33: $137-144$ 
Luangsaard JJ, Hywel-Jones NL, Manoch L, Samson RA (2005) On the relationships of Paecilomyces sect. Isarioidea species. Mycol Res 109:581-589

Nonaka K, Kaifuchi S, Omura S, Masuma R (2013a) Five new Simplicillium species (Cordycipitaceae) from soils in Tokyo, Japan. Mycoscience 54:42-53

Nonaka K, Omura S, Masuma R, Kaifuchi S, Masuma R (2013b) Three new Pochonia taxa (Clavicipitaceae) from soils in Japan. Mycologia 105:1202-1218

Patel RK, Singh A, Deka BC, Ngachan SV (2008) Handbook of fruit production. ICAR Research Complex for NEH Region, Meghalaya

Posada D (2008) jModelTest 0.1 package available at http://darwin. uvigo.es

Rambaut A (2012) FigTree 1.4.0 available at http://tree.bio.ed.ac.uk

Rambaut A, Drummond AJ (2007) Tracer version 1.5 available from http://beast.bio.ed.ac.uk

Rehner SA, Samuels GJ (1995) Molecular systematics of the Hypocreales: a teleomorph gene phylogeny and the status of their anamorphs. Can J Bot 73:816-823

Summerbell RC, Gueidan C, Schroers HJ, de Hoog GS, Starink M, Arocha Rosete Y, Guarro J, Scott JA (2011) Acremonium phylogenetic overview and revision of Gliomastix, Sarocladium, and Trichothecium. Stud Mycol 68:139-162

Sung GH, Spatafora JW, Zare R, Hodge KT, Gams W (2001) A revision of Verticillium sect. Prostrata. II. Phylogenetic analyses of SSU and
LSU nuclear rDNA sequences from anamorphs and teleomorphs of the Clavicipitaceae. Nova Hedwigia 72:311-328

Tamura K, Peterson D, Peterson N, Stecher G, Nei M, Kumar S (2011) MEGA5: molecular evolutionary genetics analysis using maximum likelihood, evolutionary distance, and maximum parsimony methods. Mol Biol Evol 28:2731-2739

Vilgalys R, Hester M (1990) Rapid genetic identification and mapping of enzymatically amplified ribosomal DNA from several Cryptococcus species. J Bacteriol 172:4238-4246

White TJ, Bruns TD, Lee S, Taylor JW (1990) Amplification and direct sequencing of fungal ribosomal RNA genes for phylogenetics. In: Innis MA, Gelfand DH, Sninsky JJ, White TJ (eds) PCR protocols: a guide to methods and applications. Academic Press, San Diego, pp 315-322

Zare R, Gams W (2004) A monograph of Verticillium section Prostrata. Rostaniha 3:1-188

Zare R, Gams W (2008) A revision of the Verticillium fungicola species complex and its affinity with the genus Lecanicillium. Mycol Res 112:811-824

Zare R, Gams W, Culham A (2000) A revision of Verticillium sect. Prostrata. I. Phylogenetic studies using ITS sequences. Nova Hedwigia 71:465-480

Zare R, Gams W, Starink-Willemse M, Summerbell RC (2007) Gibellulopsis, a suitable genus for Verticillium nigrescens, and Musicillium, a new genus for $V$. theobromae. Nova Hedwigia 85:463-489 